\title{
Alterações radiográficas e ultra-sonográficas iniciais em osteoartrite experimental eqüina
}

\author{
[Early radiographic and ultrasonographic changes in equine experimental osteoarthritis] \\ L. Rasera ${ }^{1}$, D. G. Macoris ${ }^{1}$, J. C. Canola ${ }^{1}$, A. C. Aita ${ }^{1}$, T. L. S. Gomes ${ }^{2}$ \\ ${ }^{1}$ Faculdade de Ciências Agrárias e Veterinárias - UNESP \\ Via de Acesso Prof. Paulo Donato Castelane, s/n \\ 14884-900 - Jaboticabal, SP \\ ${ }^{2}$ Hospital de Eqüinos Salles Gomes, Foz e Associados - São Paulo, SP
}

\begin{abstract}
RESUMO
Avaliaram-se oito articulações metacarpofalangeanas (MCF) de eqüinos adultos pelos exames radiográfico e ultra-sonográfico após indução cirúrgica de uma instabilidade articular, para mimetizar as alterações iniciais da osteoartrite (OA) naturalmente adquirida. Foram mensurados a circunferência articular, a amplitude do movimento articular e o grau de claudicação dos membros torácicos (avaliação clínica). Dez dias após a intervenção cirúrgica os animais foram exercitados em um andador por um período de 60 dias. Os exames radiográfico e ultra-sonográfico foram realizados antes da intervenção cirúrgica e após 25, 40 e 70 dias, e a avaliação clínica, semanalmente, durante todo o período experimental. Observaram-se alterações ultra-sonográficas aos 25 dias após a instabilidade articular, enquanto as alterações radiográficas somente foram visualizadas aos 40 dias. Houve aumento significativo $(\mathrm{P}<0,05)$ da circunferência articular da articulação MCF operada $(25,75 \mathrm{~cm} \pm 1,1)$ a partir do sétimo dia, em relação à articulação controle $(24,88 \mathrm{~cm} \pm 1,0)$ e diminuição da amplitude do movimento da articulação operada $\left(96,38^{\circ} \pm 10,7\right)$ a partir do $25^{\circ}$ dia, em relação ao seu valor basal $\left(109,24^{\circ} \pm 10,3\right)$ e em relação ao controle $\left(108,75^{\circ} \pm 8,3\right)$. $\mathrm{O}$ grau de claudicação aumentou significativamente $(\mathrm{P}<0,05)$ durante $\mathrm{o}$ período do estudo, com valor médio do escore de 1,5 para o membro operado em relação ao membro contralateral. Em conclusão, os sinais iniciais da OA cirurgicamente induzida foram melhor e mais precocemente detectados pela ultra-sonografia quando comparado ao exame radiografico. As mensurações da circunferência articular e da amplitude do movimento articular são úteis na avaliação das doenças articulares.
\end{abstract}

Palavras-chave: eqüino, articulação, osteoartrite, radiografia e ultra-sonografia

\begin{abstract}
Eight metacarpophalangeal joints (MCP) of mature horses were evaluated by radiographic and ultrasonographic exams after surgically instability-induced that mimics the early changes of naturally acquired osteoarthritis (OA). Joint circunference, range of motion and lameness score were measured (clinical evaluation). Ten days after surgically joint instability the animals were walked on a horse walker by a 60-day period. Radiography and ultrasonography were taken immediately before desmotomy and 25, 40 and 70 days after surgery. Clinical evaluation was performed at each 7-day intervals. Ultrasonographic changes were observed on day 25 after articular instability while radiographic changes were only visualized on day 40. Joint circunference was significantly higher $(P<0.05)$ in $O A$ joints $(25.75 \mathrm{~cm} \pm 1.1)$ on day 7 as compared with contralateral joints $(24.88 \mathrm{~cm} \pm 1.0)$ and a range of
\end{abstract}

Recebido em 5 de maio de 2006

Aceito em 12 de maio de 2007

E-mail: lurasera@hotmail.com 
motion was significantly less $(P<0.05)$ in $O A$ joints $\left(96.38^{\circ} \pm 10.7\right)$, on day 25 , compared with basal value (109.24 \pm 10.3$)$ and contralateral joint (108.75 \pm 8.3$)$. Lameness score significantly increased $(P<0.05)$ over time with mean score 1.5 for the 70-day study period. In conclusion, changes of induced OA joint were earlier and better detected by ultrasonography than by radiography. The measure of joint circunference and range of motion are useful in joint disease evaluation.

Keywords: equine, joint, osteoarthritis, radiography and ultrasonography

\section{INTRODUÇ̃̃̃O}

As mudanças iniciais em tecidos moles periarticulares e cartilagem articular nos processos osteoartríticos são difíceis de se diagnosticar. No caso da articulação metacarpofalangeana (MCF) de eqüinos, a anestesia regional e intra-articular, o exame físico e a termografia relacionam a claudicação a essa articulação, mas fornecem poucas informações sobre a progressão da doença (Sage e Turner, 2002). O exame radiográfico pode indicar as lesões ósseas apenas após 45 dias, as quais são conseqüentes da destruição da cartilagem articular e do envolvimento ósseo secundário. Devido a isto, as mudanças iniciais da doença degenerativa articular podem não ser observadas nas radiografias (Morgan, 1968).

A ultra-sonografia é utilizável para examinar os tecidos moles peri-articulares (Aristizábol et al., 2005), as superfícies articulares e detectar as mudanças iniciais da osteoartrite (OA). Obtêm-se informações precisas com a ultra-sonografia quando se avaliam as mudanças da membrana sinovial e da cápsula articular e o aumento de volume dos tecidos peri-articulares (Denoix et al., 1996). Além disso, a ultra-sonografia parece ser mais sensível que a radiografia para a identificação de osteófitos e análise do remodelamento peri-articular nas fases iniciais da doença, permitindo ainda uma avaliação em tempo real (Denoix, 1996; Denoix e Busoni, 1998).

No intuito de se estudar as mudanças iniciais no metabolismo e morfologia articulares, pode-se provocar a osteoartrite através da criação de uma instabilidade articular. Este método foi utilizado em coelhos e cães por Muir e Carney (1987) e, em eqüinos, por Simmons et al. (1999), que mantiveram o membro contralateral como controle para compensar as consideráveis variações que existem entre os indivíduos.
O objetivo deste estudo foi realizar os exames radiográfico e ultra-sonográfico da articulação MCF de eqüinos, segundo técnica descrita por Denoix et al. (1996), associados ao exame físico semanal com mensuração da circunferência articular e da amplitude do movimento articular, após indução cirúrgica da instabilidade articular, para se obter uma variedade de informações e medidas aplicáveis aos casos clínicos de doença articular.

\section{MATERIAL E MÉTODOS}

Utilizaram-se ambas as articulações MCF de oito eqüinos adultos, sem histórico de claudicação. Os animais foram submetidos a exames físicos diários durante 0 período pós-operatório imediato (10 dias) e, em seguida, avaliados semanalmente, durante 70 dias (basal, 7, 14, 21, $28,35,42,49,56,63$ e 70 dias).

Realizou-se a avaliação do sistema locomotor, por meio de inspeção e palpação de ambas as articulações, e a classificação da claudicação foi graduada de 0 a 4 segundos Stashak (1994). Além disso, realizou-se a mensuração da circunferência articular (em centímetros), com o uso de uma fita métrica posicionada na porção mais ampla da articulação MCF.

Os animais foram submetidos à desmotomia do colateral lateral e do sesamoideano colateral lateral da articulação MCF selecionada ao acaso, segundo técnica descrita por Simmons et al. (1999).

Após 10 dias, os animais foram submetidos a um programa de exercícios em andador. Inicialmente, foram trabalhados ao passo ( $5 \mathrm{~min} / \mathrm{dia}$; três dias/semana por duas semanas, $100 \mathrm{~m} / \mathrm{min})$ e, em seguida, ao trote $(15 \mathrm{~min} / \mathrm{dia}$; três dias/semana por seis semanas, $200 \mathrm{~m} / \mathrm{min}$ ). 
O exame ultra-sonográfico de ambas as articulações MCF foi realizado no período anterior à cirurgia e aos 25, 40 e 70 dias de pósoperatório. Fez-se ultra-sonografia nos planos longitudinais (dorsal, dorsolateral e lateral) e nos planos transversais (dorsal e lateral), na altura da articulação MCF, segundo técnica descrita por Denoix et al. (1996). Avaliaram-se os tecidos moles peri-articulares, cápsula articular, membrana sinovial, margem articular e tecido ósseo subcondral.

Também foram realizadas mensurações da cartilagem articular e cápsula articular nas imagens longitudinais sagital e parasagital e na imagem transversal dorsal e verificou-se o ligamento colateral lateral nas imagens longitudinal e transversal abaxial.

Para tanto, utilizou-se um aparelho de ultrasonografia ${ }^{1}$, com Modo-Cine e transdutor linear de 7,5MHz. As imagens e os valores das mensurações realizadas foram impressos em papel térmicos ${ }^{2}$, utilizando-se vídeo impressora ${ }^{3}$.

Realizaram-se exames radiográficos nas projeções dorsopalmar, lateromedial em apoio, lateromedial flexionada, dorsomedialpalmarolateral $45^{\circ}$ oblíqua e dorsolateralpalmaromedial $45^{\circ}$ oblíqua das articulações MCF, para avaliação de alterações ósseas e determinação da amplitude do movimento articular. As radiografias foram feitas antes da intervenção cirúrgica e aos 25, 40 e 70 dias de pós-operatório.

A avaliação das imagens radiográficas foi realizada fixando-se escores, utilizando uma escala de zero a cinco para as alterações nos tecidos ósseos $(0=$ nenhuma; $1=$ sugestivo de mineralização da margem articular; $2=$ margem articular moderadamente elevada; 3 = elevação da margem articular facilmente reconhecida; $4=$ labeamento proeminente ao redor da margem articular e $5=$ labeamento proeminente e irregular, com fragmentação ou mineralização nos tecidos subjacentes) e nos tecidos moles peri-articulares $(0=$ nenhum; $1=$ perda dos planos teciduais; 2 = leve aumento de volume ou confinado à cápsular articular; $3=$ moderada

\footnotetext{
1 Scanner 200 VET - Pie Medical

${ }^{2}$ Mitsubishi K65HM

${ }^{3}$ Mitsubishi P90W888
}

distensão capsular; 4 = distensão severa, com envolvimento de outros tecidos e $5=$ grande aumento de volume, envolvendo todos os tecidos moles peri-articulares).

Para determinar a amplitude do movimento da articulação MCF, a projeção lateromedial foi feita com o apoio do peso sobre o membro (dorsoflexão do boleto) e projeção lateromedial flexionada em máxima flexão. Nas radiografias obtidas nessas duas projeções, traçaram-se duas linhas, uma passando pelo centro do terceiro osso metacarpiano e outra pela falange proximal. Utilizando a intercessão dessas duas linhas, foi determinado o ângulo palmar entre o terceiro osso metacarpiano e a falange proximal, quando em dorsoflexão e em flexão máxima. Pela diferença entre os dois valores, obteve-se a medida da amplitude total do movimento articular, em graus (Strand et al., 1995).

Para realização dos exames radiográficos, utilizou-se um aparelho de raios-x portátil ${ }^{4}$, com a distância foco-filme mantida em $28 \mathrm{~cm}$. As películas radiográficas ${ }^{5}(24 \times 30 \mathrm{~cm})$ foram usadas com chassis rígidos ${ }^{6}$ e compostos por telas intensificadoras rápidas com emissão de luz verde $^{7}$. As películas radiográficas expostas à radiação foram identificadas e posteriormente submetidas ao processo de revelação automático $^{8}$.

Utilizou-se delineamento em blocos (animais) ao acaso, em esquema de parcelas subdivididas, tendo como parcela o fator membro (controle $\mathrm{x}$ operado) e como sub parcelas o tempo (basal, 25, 40 e 70 dias). Utilizou-se o teste Duncan para comparação entre tempos e o teste $\mathrm{t}$ de Student para comparação entre grupos dentro do mesmo tempo, no nível de 5\% de probabilidade.

Para a variável grau de claudicação utilizou-se o teste não-paramétrico de Wilcoxon para comparar os membros (controle x operado) nos diversos tempos (basal, 7, 14, 21, 28, 35, 42, 49, 56,63 e 70 dias).

\footnotetext{
${ }^{4}$ Micro X/100HD

${ }^{5}$ Kodak G/RA

${ }^{6}$ Kodak

${ }^{7}$ Kodak - Lanex Regular Screens

${ }^{8}$ Runzomatic 130 - EMB
} 


\section{RESULTADOS}

Todos os animais apresentaram aumento de volume dos tecidos moles peri-articulares no aspecto lateral da articulação operada, no local da incisão, nos primeiros dias após a desmotomia. A diminuição gradual desse aumento de volume foi observada a partir do $25^{\circ}$ dia de pós-operatório.

O grau de claudicação do membro operado aumentou significativamente $(\mathrm{P} \leq 0,05)$ a partir do sétimo dia após a intervenção cirúrgica em relação ao membro controle (escore 0), e essa diferença manteve-se durante todo o período experimental (Fig. 1).

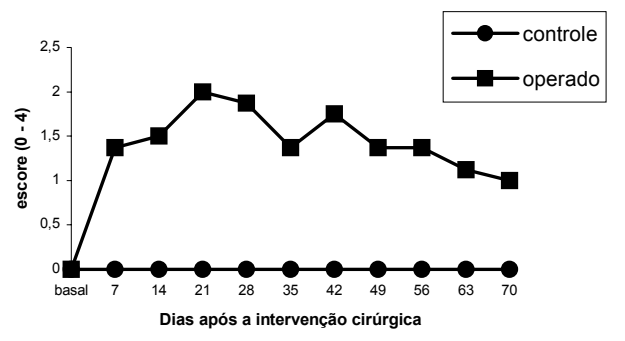

Figura 1. Variações dos valores médios do escore para grau de claudicação do membro operado de eqüino aos $7,14,21,28,35,42,49,56,63$ e 70 dias após a desmotomia do colateral lateral e do sesamoideano colateral lateral da articulação MCF. O membro controle permaneceu com grau zero de claudicação.

Houve aumento significativo $(\mathrm{P} \leq 0,05)$ na circunferência da articulação MCF operada $(25,75 \mathrm{~cm} \pm 1,1 ; \mathrm{P} \leq 0,05)$ em relação ao valor basal $(24,88 \mathrm{~cm} \pm 1,0)$ a partir do sétimo dia após a cirurgia e a partir do $14^{\circ}$ dia $(26,25 \mathrm{~cm} \pm 1,0)$ em relação ao membro controle $(24,56 \mathrm{~cm} \pm 1,0)$, mantendo-se constante até os 70 dias (Fig. 2).

A amplitude do movimento da articulação MCF operada diminuiu significativamente $\left(96,38^{\circ} \pm 10,7\right.$; $\mathrm{P} \leq 0,05)$ em relação ao valor basal $\left(109,24^{\circ} \pm 10,3\right)$, e em relação ao membro controle $\left(108,75^{\circ} \pm 8,3\right)$, a partir do $25^{\circ}$ dia de pós-operatório, diferença que permaneceu até o término do experimento (Fig. 3).

Todos os animais apresentaram aumento da espessura da cápsula articular fibrosa dorsal da articulação operada aos 25 dias $(0,83 \mathrm{~cm}) ; 40$ dias $(0,88 \mathrm{~cm})$ e 70 dias $(0,77 \mathrm{~cm})$ de pós-operatório (Fig. 4 e 5) quando comparado aos valores basais
$(0,70 \mathrm{~cm})$. Dois animais apresentaram uma linha anecóica entre a cápsula e a cartilagem articular.

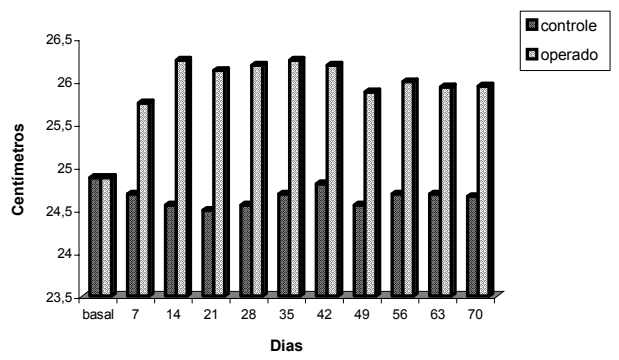

Figura 2 - Variações dos valores médios da circunferência articular $(\mathrm{cm})$ da articulação MCF controle e operada de eqüino aos 7, 14, 21, 28, 35, 42, $49,56,63$ e 70 dias após a desmotomia do colateral lateral e do sesamoideano colateral lateral.

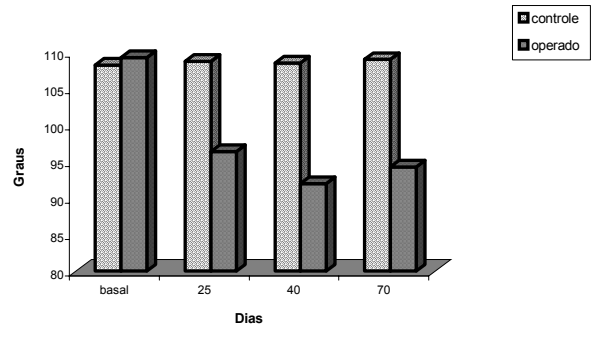

Figura 3 - Variações dos valores médios da amplitude do movimento articular (graus) das articulações MCF controle e operada de eqüino aos 25,40 e 70 dias após a desmotomia do colateral lateral e dos sesamoideano colateral lateral.

Observou-se diminuição na espessura da cartilagem articular aos 25 dias, em todos os animais, com dificuldades na visualização desta nas imagens ultra-sonográficas (Fig. 4 e 5). A superfície óssea subcondral apresentou irregularidades no côndilo lateral do terceiro osso metacarpiano aos 25 dias após a intervenção cirúrgica.

A transecção do ligamento colateral lateral foi evidente no exame ultra-sonográfico, apresentando imagem hipoecóica e acúmulo de material anecóico em seu interior, em todos os animais. Após 70 dias da intervenção cirúrgica houve aumento na espessura do ligamento colateral lateral em suas camadas superficial e profunda. 


\section{Rasera et al.}

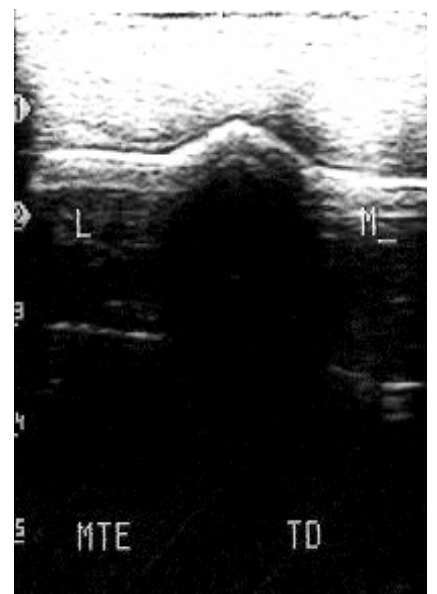

A

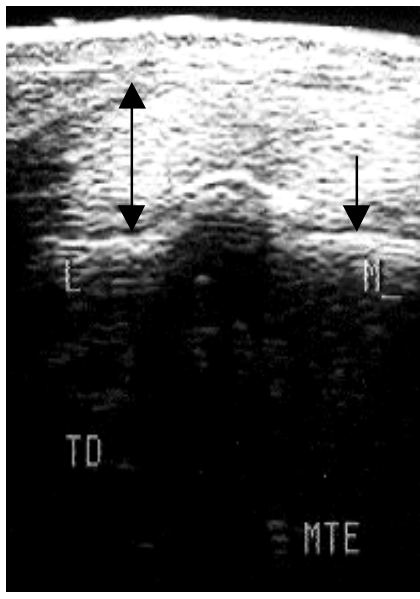

C

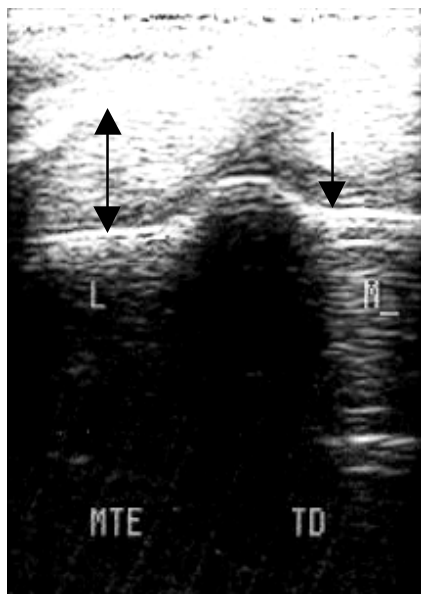

B

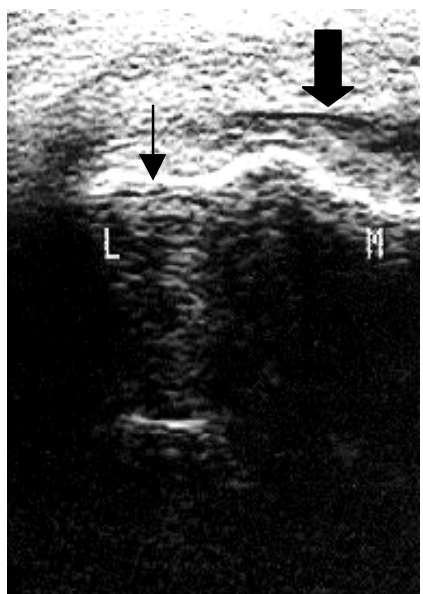

$\mathrm{D}$

Figura 4. Imagens ultra-sonográficas transversais dorsais da articulação MCF de eqüino antes (A) e aos 25 (B), 40 (C) e 70 (D) dias após a desmotomia do colateral lateral e sesamoideano colateral lateral, mostrando o espessamento da cápsula articular dorsal (seta dupla) e a diminuição da espessura da cartilagem articular (B, C e D) (seta fina). Presença de material anecóico na cápsula articular aos 70 dias (D) (seta grossa).

As lesões ósseas radiográficas foram menos evidentes, sendo necessária a magnificação da imagem (Tab. 1). Não foram observadas esclerose do osso subcondral, diminuição do espaço articular e presença de osteófitos nas articulações MCF estudadas, durante o período analisado.

Tabela 1. Escores das imagens radiográficas obtidas da articulação metacarpofalangeana após desmotomia do colateral lateral e sesamoideano colateral lateral

\begin{tabular}{ccc}
\hline \multirow{2}{*}{ Dias } & \multicolumn{2}{c}{ Achados radiográficos } \\
\cline { 2 - 3 } & Alterações nos tecidos ósseos & $\begin{array}{c}\text { Alterações nos tecidos moles } \\
\text { peri-articulares }\end{array}$ \\
\hline 0 & 0 & 0 \\
25 & 0 & $1^{*}$ \\
40 & 1 & $1^{*}$ \\
70 & 1 & 0 \\
\hline
\end{tabular}

*Alterações nos tecidos moles somente no aspecto abaxial da articulação MCF. 
A amplitude do movimento da articulação MCF operada diminuiu significativamente $\left(96,38^{\circ} \pm 10,7 ; \mathrm{P} \leq 0,05\right)$ em relação ao valor basal $\left(109,24^{\circ} \pm 10,3\right)$, e em relação ao membro controle $\left(108,75^{\circ} \pm 8,3\right)$, a partir do $25^{\circ}$ dia de pós-operatório, diferença que permaneceu até o término do experimento (Fig. 5).

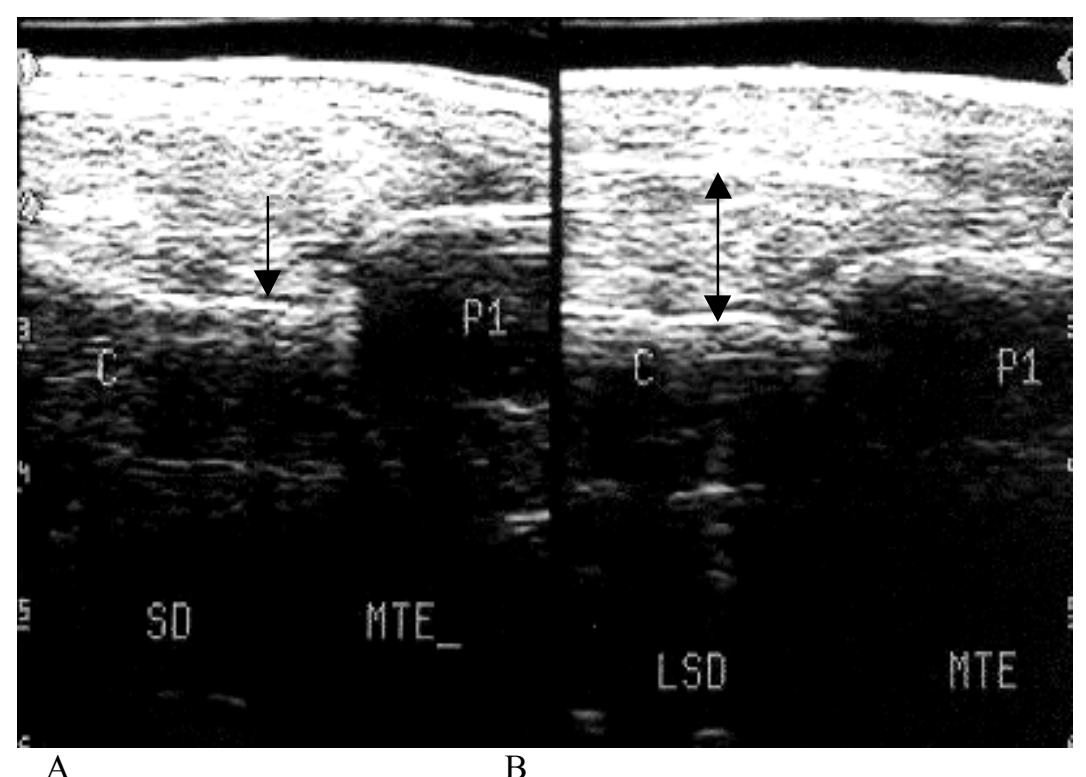

Figura 5. Imagens ultra-sonográficas longitudinal dorsal (A) e dorsolateral (B) da articulação MCF de eqüino aos 25 dias após a desmotomia do colateral lateral e do sesamoideano colateral lateral, mostrando a diminuição da espessura da cartilagem articular (A) (seta fina) e o espessamento da cápsula articular (B) (seta dupla).

\section{DISCUSSÃO}

O modelo de instabilidade articular preconizado por Simmons et al. (1999) origina os processos degenerativos e os sinais clássicos da OA, como claudicação, aumento da circunferência articular e diminuição da amplitude do movimento articular. A claudicação, em casos agudos, ocorre devido à dor, a qual pode estar relacionada às lesões do ligamento colateral lateral e cápsula articular, assim como restrição mecânica devido à fibrose nos estágios crônicos da doença (McIlwraith, 1994; Simmons et al., 1999). Neste estudo, os animais apresentaram um grau leve de claudicação nos primeiros dias de pós-operatório, sendo que este foi diminuindo durante o período experimental com tendência à estabilização, o que é característico da $\mathrm{OA}$ naturalmente adquirida.

Durante as fases iniciais do período experimental houve aumento da circunferência articular relacionado ao espessamento da cápsula articular e à efusão sinovial devido ao trauma cirúrgico, diminuindo nas últimas semanas, confirmando os achados de Muir e Carney (1987). O edema dos tecidos moles peri-articulares e a restrição mecânica pela fibrose contribuíram para a diminuição da amplitude do movimento articular nas articulações operadas em relação ao controle, durante todo o período analisado. Estes sinais podem ser importantes para avaliar as mudanças que ocorrem na cápsula articular fibrosa dos cavalos com atividade atlética e que apresentam doença articular.

O exame ultra-sonográfico demonstrou aumento na espessura da cápsula articular dorsal, diminuição na espessura da cartilagem articular, evidenciada pela falta de regularidade em toda a superfície e dificuldade de sua visualização na imagem ultra-sonográfica, e irregularidade da margem articular aos 25 dias de pós-operatório. A diminuição na espessura da cartilagem e sua irregularidade são mudanças características nas 
articulações com osteoartrite (Tomlinson et al., 2000).

A cronologia das alterações radiográficas nos tecidos ósseos sugere que o exame radiográfico para o diagnóstico de doença articular poderá ser repetido aos 40 dias após o primeiro exame. As mudanças iniciais da osteoartrite não são bem documentadas pelo exame radiográfico e aparecem tardiamente como resultado da destruição da cartilagem articular e envolvimento ósseo secundário, quando as erosões na cartilagem são mais severas e o espaço articular torna-se diminuído (Morgan, 1968; Rooney, 1969), demonstrado neste estudo pela visualização das alterações ósseas somente com 40 dias do início da instabilidade articular.

Todos estes achados sugerem que a ultrasonografia deve ser feita em cavalos com doença articular e que se apresentam radiograficamente normais. Os cavalos que são submetidos à cirurgia articular devem ser examinados ultrasonograficamente no período pós-operatório, e o exame radiológico deve ser repetido aos 40 dias após a cirurgia, o que não é indicado por alguns autores (Murphy e Nixon, 2001; Elce e Richardson, 2002). Adicionalmente, o acompanhamento por mensuração da circunferência articular e da amplitude do movimento das articulações dos cavalos atletas nos permite avaliar as mudanças clínicas iniciais que ocorrem nos tecidos articulares devido ao trauma repetitivo.

Em conclusão, o modelo de instabilidade articular associado ao exercício produziu osteoartrite na articulação MCF e as alterações iniciais foram melhor e mais precocemente detectadas pela ultra-sonografia quando comparada ao exame radiográfico, e sua aplicação clínica para exame das claudicações com origem na articulação MCF pode revelar lesões não aparentes radiograficamente. Além disso, a mensuração da circunferência articular e a da amplitude do movimento são úteis na avaliação das doenças articulares.

\section{AGRADECIMENTOS}

Aos Doutores Cláudia Acosta Duarte e Stefano Hagen pela colaboração.

\section{REFERÊNCIAS BIBLIOGRÁFICAS}

ARISTIZÁBEL, M.; SOUZA, M.V.; ARANZALES, J.R.M. et al. Valores biométricos obtidos por ultra-sonografia dos tendões flexores e ligamentos acessório inferior e suspensório da região metacárpica palmar de cavalos Mangalarga Marchador. Arq. Bras. Med. Vet. Zootec., v.57, p. 156-162, 2005

DENOIX, J.M. Ultrasonographic examination in the diagnosis of joint disease. In: McILWRAITH, C.W.; TROTTER, G. W. Joint disease in the horse. Philadelphia: Saunders, 1996. p 40-70.

DENOIX, J.M.; STEPHANIE, J.; BOUSSEAU, B. et al. Ultrasonographic anatomy of the dorsal and abaxial aspects of the equine fetlock. Equine Vet. J.,v.28, p.54-62, 1996.

DENOIX, J.M.; BUSONI, V. Ultrasonography of joint and synovia. In: WHITE II, N.A.; MOORE, J.N. Current techniques in equine surgery and lameness. Philadelphia: Saunders, 1998. p.643-654.

ELCE, A.Y.; RICHARDSON, D.W. Arthroscopic removal of dorsoproximal chip fractures of the proximal phalanx in standing horses. Vet. Surg., v.31, p.195-200, 2002.

McILWRAITH, C.W. Doenças das articulações, tendões, ligamentos e estruturas relacionadas. In: STASHAK, T.S. Claudicação em eqüinos segundo Adam's. 4.ed. São Paulo: Rocca, 1994. p.350-502.

MORGAN, J.P. Radiographic diagnosis of bone and joint diseases in the horse. Cornell Vet., v.58, p.28-47, 1968.

MUIR, H.; CARNEY, S.L. Pathological and biochemical changes in cartilage and other tissues of the canine knee resulting from induced joint instability. In: HELMINEN, H.I.; KIVIRANTA, I.; TAMMI, M. et al. Joint loading biology and health of articular structures. Bristol: Wright, 1987. p.149-186.

MURPHY, D.J.; NIXON, A.J. Arthroscopic laser extirpation of metacarpophalangeal synovial pad proliferation in eleven horses. Equine Vet. J., v.33, p.296-301, 2001.

ROONEY, J.R. Biomechanics of lameness in horses. Baltimore: Williams \& Wilkins, 1969. p.69-78, 162-180.

SAGE, A.M.; TURNER, T.A. Ultrasonography of the soft tissue structures of the equine foot. Equine Vet. Educ., v.14, p.212-221, 2002.

SIMMONS, E.J.; BERTONE, A.J.; WEISBRODE, S.E. Instability-induced osteoarthritis in the metacarpophalangeal joint of horses. Am. J. Vet. Res., v.60, p.7-13, 1999.

STASHAK, T.A. Relação entre conformação e claudicação. In:_ Claudicação em eqüinos segundo Adam's. 4.ed. São Paulo: Rocca, 1994. p.108-109.

STRAND, E.; MARTIN, G.S.; CRAWFORD, M.P. et al. Intra-articular pressure, elastance, and range of motion in flexion of the equine metacarpophangeal joint. Am. J. Vet. Res., v.56, p.1362-1371, 1995.

TOMLINSON, J.E.; REDDING, W.R.; SAGE, A.M. Ultrasonographic evaluation of arsocrural joint cartilage in normal adult horses. Vet. Rad. Ultr.,v.41, p.457-460, 2000. 